\title{
Investing for Reliability and Security in Transportation Networks
}

\author{
Lei Zhang and David Levinson
}

\begin{abstract}
Alternative transportation investment policies can lead to very different network forms in the future. The desirability of a transportation network should be assessed not only by its economic efficiency but also by its reliability and security, because the cost of an incidental capacity loss in a road network can be massive. This research concerns how investment rules shape the hierarchical structure of roads and affect network fragility to natural disasters, congestion, and accidents and vulnerability to targeted attacks. A microscopic network growth model predicts the equilibrium road networks under two alternative policy scenarios: investment based on benefit-cost analysis and investment based on bottleneck removal. A set of Monte Carlo simulation runs, in which a certain percentage of links was removed according to the type of network degradation analyzed, was carried out to evaluate the equilibrium road networks. It was found that a hierarchy existed in road networks for reasons such as economic efficiency but that an overly hierarchical structure had serious reliability problems. Throughout the equilibrating or evolution process, the grid network studied under benefit-cost analysis had better efficiency performance, as well as error and attack tolerance. The paper demonstrates that reliability and security considerations can be integrated into the planning of transportation systems.
\end{abstract}

Transportation networks support various vital human activities, and their performance affects the efficiency of virtually all economic transactions. The design of an effective transportation network has thus become an important task for planners. Traditionally, the desirability of a transportation network is assessed by its efficiency under prevailing conditions, for example, the total travel cost of moving a subject from an origin to a destination. More recently, several measures of transportation network reliability have explicitly considered the probabilistic nature of network performance. The uncertainty may be created by one or more of the following sources: demand fluctuation, random link failures because of congestion or accidents, natural disasters, or targeted attacks. Decision makers need to consider the potential trade-off between network efficiency and reliability when they forge policies that could shape future transportation networks.

For several economic and geographical reasons, transportation networks typically evolve into hierarchical structures in which few links carry the bulk of traffic and a large number of smaller links have very low flows. A subsequent question is whether such a hierarchical struc-

L. Zhang, School of Civil and Construction Engineering, Oregon State University, 220 Owen Hall, Corvallis, OR 97331. D. Levinson, Department of Civil Engineering, University of Minnesota, 500 Pillsbury Drive SE, Minneapolis, MN 55455. Corresponding author: L. Zhang, lei.zhang@oregonstate.edu.

Transportation Research Record: Journal of the Transportation Research Board, No. 2041, Transportation Research Board of the National Academies, Washington, D.C., 2008, pp. 1-10.

DOI: 10.3141/2041-01 ture is susceptible to random failures (fragility) and targeted attacks (vulnerability). More importantly, the desire is to know the kinds of policies that tend to create a network form that is neither fragile nor vulnerable. These research objectives distinguish this research from previous studies that have focused on the measurement of network reliability (1-5) and the identification of weak spots in existing networks (6). Compared with the attempts that have been made to include reliability measures in the network design problem (7), the models presented below simulate the long-term impacts of the repetitive execution of an investment policy over a period of time.

This paper develops a model of transportation network growth under alternative policies at the link (microscopic) level. Two road investment rules, benefit-cost analysis and bottleneck removal, are modeled, and the performance of the resulting road networks at equilibrium is compared. The robustness and reliability of the equilibrium networks are evaluated under several network degradation scenarios, including random link failures and targeted attacks, followed by conclusions and suggestions for future research.

\section{MODEL OF TRANSPORTATION NETWORK GROWTH}

The network dynamics model specified in this section brings together all relevant agents and their interactions to simulate road expansion and contraction. The complexity of the whole transportation system in a region cannot be described fully by the proposed transportation network growth model, and therefore, it has certain limitations. Economic growth must be taken as an exogenous variable, because the transportation infrastructure is not the only factor that drives economic growth. Land use dynamics and population changes are also treated as exogenous variables in the network analysis. These limitations can be removed in future research. Attention is focused on road network growth, a process with enough complicated and unknown dynamics for one to start with. The endogenous variables considered in the model are travel behavior and demand, road maintenance and expansion costs, pricing rules and revenue, investment policy, and road expansion and degeneration.

An overview of the model components and their interconnectivity is shown in Figure 1. A travel demand model predicts link-level flows on the basis of the road network, land use patterns, and socioeconomic and demographic information. On the basis of the demand forecasting results, links are used to calculate revenues and costs. An investment module then operates and causes annual supply changes, producing an updated network. The modeling process does not have to iterate annually. Other updating intervals can also be used. Yearly supply changes, however, correspond to budgets that are typically decided each fiscal year. The road network is represented by a directed graph that connects nodes with directional arcs (links). The standard notation convention for directed graphs is adopted for the following 


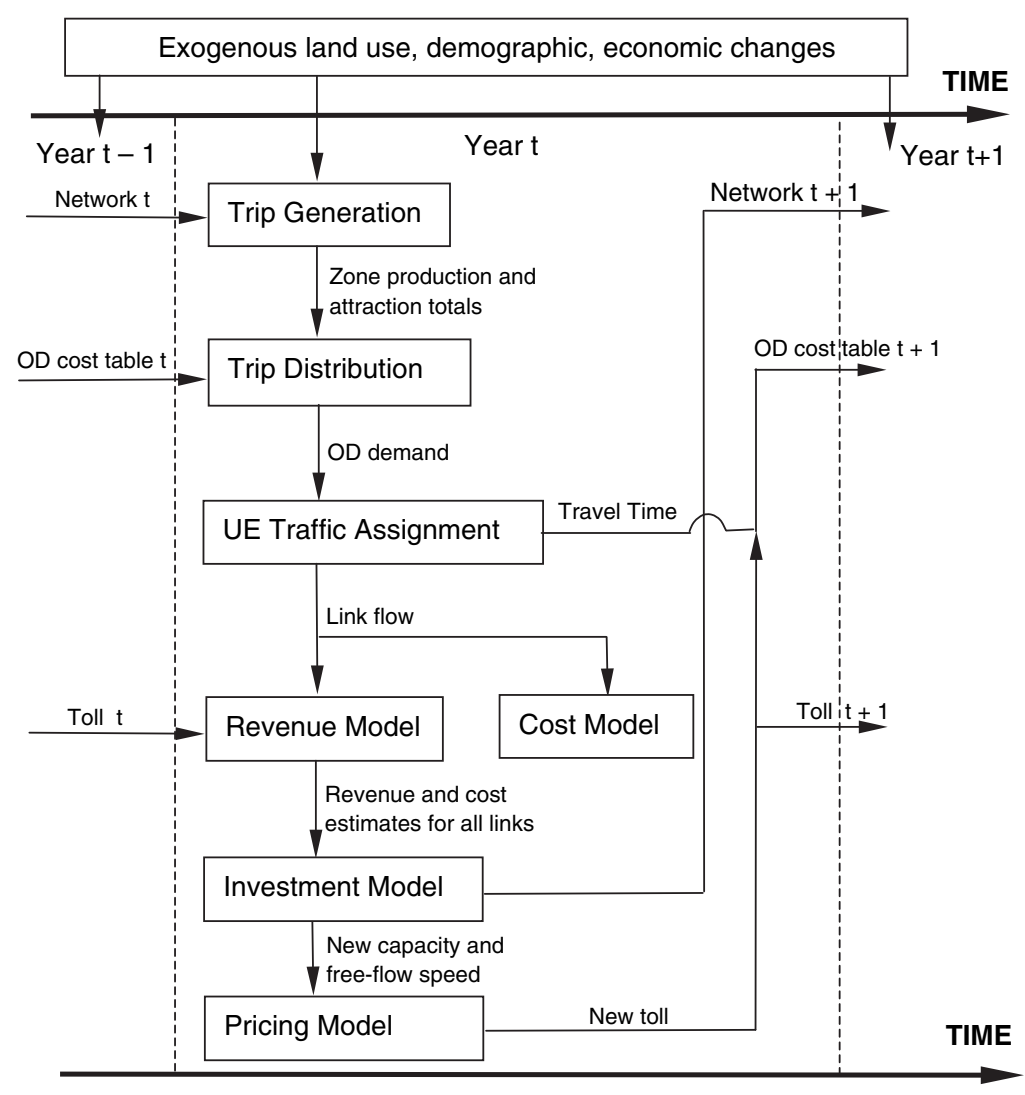

FIGURE 1 Flowchart of the network growth model (UE = user equilibrium).

presentation of the details of the mathematical formulations of the submodels.

\section{Travel Demand}

A traditional four-step forecasting model is used to predict travel demand at the link level by taking land use, socioeconomics, and the existing network as exogenous variables. A zone-based regression structure is used for trip generation. The origin-destination (O-D) cost table obtained from the traffic assignment for the previous year is used for trip distribution in the current year, based on a doubly constrained gravity model (8):

$q_{r s}^{i}=m_{r} O_{r} n_{s} D_{s} \cdot d\left(t_{r s}^{i}\right)$

where

$q_{r s}^{i}=$ demand from origin zone $r$ to destination zone $s$ in year $i$,

$O_{r}=$ number of trips produced from zone $r$,

$D_{s}=$ number of trips destined for zone $s$,

$m_{r}, n_{s}=$ coefficients in the gravity model,

$t_{r s}^{i}=$ generalized travel cost of traveling from zone $r$ to $s$, and

$d(\cdot)=$ travel cost impedance function in the gravity model, where $d\left(t_{r s}^{i}\right)=e^{-\gamma \cdot t_{r s}^{i}}$ and $\gamma$ is the coefficient in the impedance function.

The resulting O-D table is loaded onto the transportation network for the current year through the origin-based user equilibrium traffic assignment algorithm developed by Bar-Gera and Boyce (9). The generalized link cost function comprises two parts, a travel time component and a vehicle toll. The travel time component uses the Bureau of Public Roads (BPR) (10) functional form:

$t_{a}^{i}=\lambda \frac{l_{a}}{v_{a}^{i}}\left[1+\theta_{1}\left(\frac{f_{a}^{i}}{F_{a}^{i}}\right)^{\theta_{2}}\right]+\tau_{a}^{i}$

where

$t_{a}^{i}=$ generalized travel cost on link $a$ in year $i$,

$\lambda=$ value of travel time constant (dollars per hour),

$v_{a}^{i}=$ free-flow speed of link $a(\mathrm{~km} / \mathrm{h})$ in year $i$,

$F_{a}^{i}=$ capacity of link $a$ in year $i$ (number of vehicles per hour),

$l_{a}=$ length of link $a$ (constant) $(\mathrm{km})$,

$f_{a}^{i}=$ average hourly flow on link $a$ in year $i$ (number of vehicles per hour),

$\theta_{1}, \theta_{2}=$ coefficients of the BPR travel time function, and

$\tau_{a}^{i}=$ link toll per vehicle (dollars; see Equations 3 and 4 for details).

In the traffic assignment step, if the relative excess travel cost is less than 0.001, the Wardrop user equilibrium is considered to be satisfied.

\section{Price and Revenue}

Revenue is collected at the link level by means of a toll on vehicles. The annual revenue is simply the product of the toll and the annual 
flow. The amount of the toll depends on the pricing policy specified. Fuel taxes in the United States are modeled in Equation 4. Fuel taxes are essentially a distance-based toll if the variation in automobile fuel efficiency is ignored. The revenue collected from fuel taxes is assumed to be controlled by a centralized agency that also makes investment decisions.

$$
\begin{aligned}
& E_{a}^{i}=\tau_{a}^{i} \cdot\left(\psi \cdot f_{a}^{i}\right) \\
& \tau_{a}^{i}=\rho_{1} \cdot\left(l_{a}\right)^{\rho_{2}} \cdot\left(v_{a}^{i}\right)^{\rho_{3}}
\end{aligned}
$$

where

$$
\begin{aligned}
E_{a}^{i}= & \text { revenue (earnings) of link } a \text { in year } i \text { (dollars), } \\
\psi= & \text { coefficient used to scale the average hourly flow to the } \\
& \text { annual flow, and } \\
\rho_{1}= & \text { scale coefficient related to the toll level (dollars } \left.\cdot \mathrm{h}^{\rho 3} / \mathrm{km}^{\rho 2+\rho 3}\right), \\
& \text { where } \rho_{2} \text { and } \rho_{3} \text { are coefficients indicating economies or } \\
& \text { diseconomies of scale. }
\end{aligned}
$$

\section{Maintenance Cost}

Empirical evidence shows that the costs of maintaining and operating roads are only partially (30\% and $46 \%$, respectively) related to the traffic volume on the road (11; W. D. O. Paterson and R. Archondo-Callao, Estimating Road Use Cost, unpublished document, World Bank, Washington, D.C., Sept. 1991). Some maintenance expenditures are fixed and irrespective of traffic. Nonlinear increases in maintenance costs with respect to traffic volume have also been observed. Traffic loading characteristics also affect costs for pavement resurfacing. A simplified link maintenance cost function is specified with three determining factors: link length, capacity, and volume. Therefore, all maintenance costs unrelated to traffic are attributed to capacity in this model.

$M_{a}^{i}=\mu \cdot\left(l_{a}\right)^{\alpha_{1}}\left(F_{a}^{i}\right)^{\alpha_{2}}\left(f_{a}^{i}\right)^{\alpha_{3}}$

where

$$
\begin{aligned}
M_{a}^{i}= & \text { cost of maintaining link } a \text { at its present condition in } \\
& \text { year } i \text { (dollars), } \\
\mu= & \text { scale parameter (dollar } \left.\cdot \mathrm{h}^{\alpha 2+\alpha 3} / \mathrm{km}^{\alpha 1}\right) \text {, and } \\
\alpha_{1} \text { to } \alpha_{3}= & \text { coefficients indicating economies or diseconomies of } \\
& \text { scale. }
\end{aligned}
$$

\section{Construction Cost}

The cost of road construction depends on many factors: the lane miles of construction, road hierarchy (road types, e.g., Interstate highway or state highway), land acquisition cost, degree of urbanization, terrain, and elevated sections (e.g., interchanges and bridges). Keeler and Small developed a construction cost model with only two variables, lane miles of construction and degree of urbanization (12). Their model explains about $52 \%$ of the total cost variation. A regression model developed for construction projects in the Twin Cities in Minnesota is able to explain $77 \%$ of the cost variation with two additional variables: road hierarchy and the duration of construction (13). A three-variable function is specified for road construction cost. The product of the first and third terms in this cost function is similar to the lane mile variable in previous studies. The second term implies road hierarchy. According to this cost function, it is more expensive to construct a road that is longer, has a high existing capacity (is higher in the hierarchy of roads), and needs a larger capacity increase.

$K_{a}^{i}=\phi \cdot\left(l_{a}\right)^{\sigma_{1}} \cdot\left(F_{a}^{i}\right)^{\sigma_{2}} \cdot\left(F_{a}^{i+1}-F_{a}^{i}\right)^{\sigma_{3}}$

where

$$
\begin{aligned}
K_{a}^{i}= & \text { cost of expanding link } a \text { in year } i \text { (dollars), } \\
\phi= & \text { scale parameter, and } \\
\sigma_{1} \text { to } \sigma_{3}= & \text { coefficients indicating economies or diseconomies of } \\
& \text { scale. }
\end{aligned}
$$

Some consider investments in road construction to be discrete, highly indivisible, and lumpy, whereas others argue that investments in capacity improvements are usually less lumpy than they seem (14). Existing evidence supports both arguments. A road can be built with only a discrete number of lanes or can be expanded only by the addition of a discrete number of lanes. However, incremental capacity improvements can also be achieved by enhancing management efficiencies, widening shoulders, and resurfacing pavement. Equation 6 is a continuous function. However, it can also describe the costs of discrete road expansions with an additional constraint. That can be done by keeping track of the existing number of lanes for each road in the network and estimating a capacity-number-of-lane function. The capacity-number-of-lane function determines the capacity of a road section according to the number of lanes $(L)$ :

$F_{a}^{i}=\eta_{0}+\eta_{1} \cdot L_{a}^{i}+\eta_{2} \cdot L_{a}^{i^{2}}$

where $L_{a}^{i}$ is the number of lanes in link $a$ in year $i$ and $\eta_{0}$ to $\eta_{2}$ are empirically derived coefficients. The second-order term is included to capture the possible nonlinearity of capacity increases. This function can estimate the construction cost required to add one or two more lanes to an existing road. It should be noted that for simplicity the level of urbanization (indicated by residential density or the distance from the nearest downtown) is not included in the expansion cost model.

\section{Investment Rule}

The investment model takes the revenues and costs on all links as inputs and determines how revenues are distributed to maintain and expand the network. If the amount of revenue distributed to a link is not sufficient to defray its maintenance cost, the capacity of the link will decrease in the next iteration. Revenues may also be used to expand some links in the network, depending on the specific investment policy and organizational structure. The next section describes two common investment policies in detail. The amount of capacity change can be determined by the construction cost function in a discrete or continuous manner. A capacity change on a link is sometimes associated with a concurrent change in free-flow speed. Vehicles are, in general, able to travel faster on wider roads. A loglinear function is specified to capture the coevolution of capacity and free-flow speed:

$v_{a}^{i+1}=\omega_{1}+\omega_{2} \cdot \ln \left(F_{a}^{i+1}\right)$

where $\omega_{1}$ and $\omega_{2}$ are empirically derived coefficients.

With an updated link capacity and free-flow speed, some factors influencing travel behavior, such as link travel time and toll level, will change. These supply shifts, combined with preference, economic 
growth, and demographic changes, give rise to the emergence of a new demand pattern in the next iteration.

\section{Estimation of Model Parameters}

The exact values of the parameters in the network dynamics model depend on the road network in question. This section discusses in general the empirical data required for model estimation and their likely sources and, in particular, discusses the derivation of a complete set of parameter estimates for the road network in the Twin Cities metropolitan area. An earlier version of the network dynamics model was previously applied to the Twin Cities road network (L. Zhang and D. Levinson, A model of the rise and fall of roads, unpublished paper, 2008).

The first set of data required for the execution of the network dynamics model is the initial network status, including the capacity, length, free-flow speed, and tolls (if any) for all links and their connectivity. Changes in land use, demographics, and regional economy are assumed to be exogenous and, therefore, should be obtained from corresponding forecasting models.

Travel demand forecasting has become a fairly standard planning exercise over the years. Most metropolitan areas update their travel demand models, mostly four-step models, every 5 or 10 years. The parameters related to travel demand, such as $\gamma$ in the gravity model, should be available from metropolitan planning organizations (Equation 1). In the Twin Cities, the value of $\gamma$ is about 0.1 .

As mentioned earlier, the equation determining the amount of toll on a road should be derived from the specific pricing policy. In the Twin Cities, as well as other cities in the United States, fuel tax revenues have been the major sources of road funds. If the variation in gas mileage among vehicles is ignored, fuel tax is essentially a distancebased user fee $\left(\rho_{2}=1\right.$ and $\rho_{3}=0$; see Equation 4$)$. The constant term is determined from the amount of surcharge per gallon and is then normalized to unity by constant terms in the cost functions.

Keeler and Small estimated a simple linear model of road maintenance costs using project-level data (12). In their specification, maintenance cost is a function of vehicle miles of travel. Heggie calculated maintenance costs on the basis of both vehicle kilometers of travel and road hierarchy (15). Paterson and Archondo-Callao show that only about $46 \%$ of total maintenance costs are traffic related, whereas the remaining $54 \%$ of costs are fixed costs (11). Road-specific data would enable the most accurate estimation of maintenance cost. There is evidence of substantial economies of scale in maintenance cost as the traffic volume increases. The maintenance cost per vehicle kilometer of travel is much lower on a high-volume road. This suggests that $\alpha_{3}$ is between 0 and 1 (Equation 5). Although it is conceivable that higher-level roads, such as an Interstate highway, are more expensive to maintain than lower-level roads, such as an arterial street, even if they carry the same amount of traffic $\left(\alpha_{2}>0\right)$, no previous study has exactly estimated $\alpha_{2}$. It is assumed that there are diseconomies of scale in road maintenance as the capacity of the road increases $\left(\alpha_{2}>1\right)$.

For the estimation of the road expansion cost function (Equation 6), data at the project level are again the most desirable. Several previous studies on economies of scale in road construction use lane miles of expansion as a predictor, effectively assuming that $\sigma_{1}$ is equal to $\sigma_{3}(12,16,17)$. Construction projects of all sizes are combined, and therefore, it is not surprising most of them find nearly constant returns to scale $\left(\sigma_{1}=\sigma_{3}=1\right)$. A recent study with data from 110 projects in the Twin Cities showed significant increasing returns to scale $\left(\sigma_{1}=\sigma_{3}=0.5\right)$ after controlling for road hierarchy (13).
Using the same data set, the authors found constant returns to scale without controlling for road hierarchy. It should be more expensive to expand a road that already has a high capacity because of increasing land acquisition costs (13). Therefore, a $\sigma_{2}$ value $>1$ is a plausible assumption with the absence of any definitive knowledge. These observations suggest that $\sigma_{1}$ is equal to $\sigma_{3}$, which is equal to 1 , and so far seem to be the most reliable estimates for the road expansion cost function and that there is a need for further studies on cost functions (e.g., a function that considers second-order effects, factor prices, and land acquisition costs). The cost data for the Twin Cities were collected from the Transportation Improvement Program managed by the Metropolitan Council.

The database that the Metropolitan Council developed for the regional transportation planning model also includes capacity, free-flow speed, and the number of lanes for more than 10,000 road segments in the Twin Cities. These data are used to estimate the capacity-number-of-lane function (Equation 7) and the capacityspeed function (Equation 8). All coefficients are statistically significant at the .05 level $\left(\eta_{1}=341, \eta_{1}=162, \eta_{2}=273, \omega_{1}=-30.6, \omega_{2}=9.8\right)$. The $R^{2}$ values of the two models are .6 and .7 , respectively. The predicted values of the dependent variables are plotted against the observed data in Figure 2.

For normative applications (e.g., policy evaluation), the investment rule should be specified in accordance with the investment policy being evaluated. In that case, no calibration or validation is required for the investment model. The complete set of model

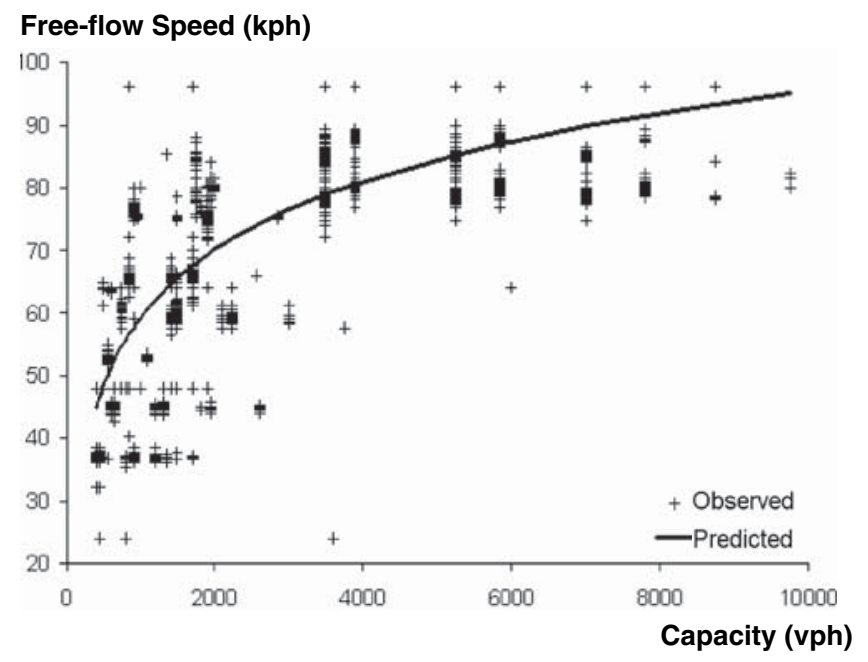

Capacity (vph)

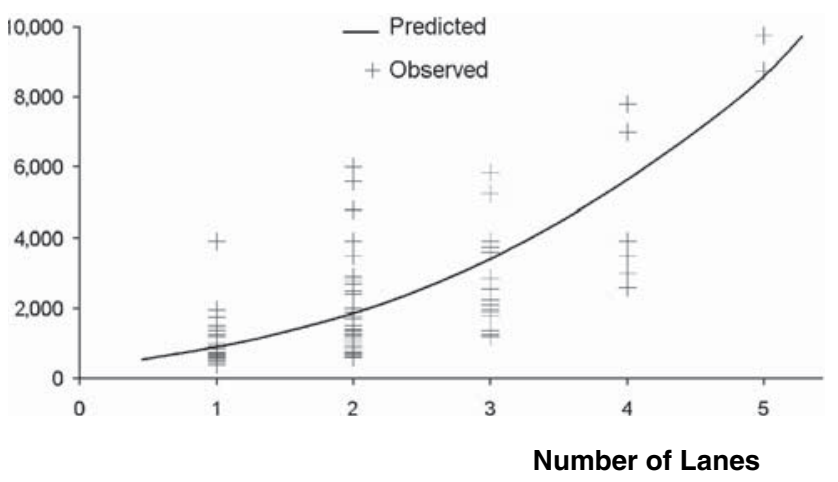

FIGURE 2 Capacity-speed (vph = number of vehicles per hour) and capacity-number-of-lane functions. 
parameters estimated or assumed for the Twin Cities (TC) road network is summarized in Table 1.

\section{INVESTMENT POLICIES}

The capacity of a road is at the optimal level if the marginal benefit of building an additional unit of capacity just equals the total cost of providing it. This optimality condition establishes a long-run investment goal but does not provide guidelines for long-term investment decisions. Benefit-cost analysis has become the basis for many investment decisions. Some engineering procedures, such as bottleneck removal, have also been established to distribute road funds to individual projects. These two investment rules are discussed and modeled for general road networks in this section.

\section{Bottleneck Removal}

The bottleneck removal policy tends to favor expansion projects on road sections with the most severe congestion problems. A mathematical model is developed to describe this type of investment rule. In this model, the total network revenue $\left(E_{T}\right)$ collected on all roads is managed by a centralized agency, which is also responsible for its distribution.

$E_{T a}^{i}=\sum_{a} E_{a}^{i}=\sum_{a} \tau_{a}^{i} \cdot\left(\psi \cdot f_{a}^{i}\right)$

All roads must be maintained at their current level of service. Total maintenance costs $\left(M_{T}\right)$ can be derived as follows:

$M_{T a}^{i}=\sum_{a} M_{a}^{i}=\sum_{a} \mu \cdot\left(l_{a}\right)^{\alpha_{1}}\left(F_{a}^{i}\right)^{\alpha_{2}}\left(f_{a}^{i}\right)^{\alpha_{3}}$

A portion of the revenue is appropriated to defray maintenance costs. The remaining disposable revenue $\left(E_{D}\right)$ is used to expand capacity on the basis of the current level of congestion, as indicated by the volume-to-capacity ratio $(S)$ on each road. Road $a$, which has the highest value of $S$ in the network, is expanded first. The amount of capacity improvement is determined by the desirable value of $S$ or the target level of service, $S^{*}$. The value of $S^{*}$ should depend on the performance of the network as a whole. The average value of $S$ for all roads is used.

$f_{a}^{i} / F_{a}^{i+1}=S^{*} \Rightarrow F_{a}^{i+1}=f_{a}^{i} / S^{*}$

$S^{*}=\sum_{a} S_{a} / N$

The required expansion cost and the remaining revenue after expansion are as indicated in Equations 13 and 14, respectively:

$K_{a}^{i}=\phi \cdot\left(l_{a}\right)^{\sigma_{1}} \cdot\left(F_{a}^{i}\right)^{\sigma_{2}} \cdot\left(F_{a}^{i+1}-F_{a}^{i}\right)^{\sigma_{3}}$

$E_{D}^{i}=E_{D}^{i}-K_{a}^{i}$

The second most congested road is expanded next. The amounts of the capacity improvements, expansion costs, and the remaining revenue can be determined similarly. This process continues until the revenue is exhausted.

One assumption in the procedure described above is the divisibility of road investments. In Equation $11, F_{a}^{i+1}$ can take any positive value. A more realistic model would allow only discrete road expansion. This assumption is relaxed by using Equation 7. In the discrete case, the desirable new capacity is compared with the actual capacity if one, two, or three lanes are added, which determines the number of lanes in the expansion and the resulting expansion costs.

\section{Benefit-Cost Analysis}

Different from bottleneck removal, benefit-cost analysis considers benefits as well as costs. It is important to establish a point of view

\section{TABLE 1 Coefficients in Network Growth Model}

\begin{tabular}{|c|c|c|c|}
\hline Parameter & Description & Values & Source \\
\hline$\lambda$ & Value of travel time constant $(\$ / \mathrm{h})$ & 10 & Empirical estimate in TC \\
\hline$\theta_{1}, \theta_{2}$ & Coefficients in the BPR function & $0.15,4$ & BPR recommended value \\
\hline$\gamma$ & Coefficient in the gravity model & 0.1 & Empirical estimate in TC \\
\hline$\rho_{1} \cdot \psi$ & Combined scale coefficient in revenue model $\left(\right.$ dollar $\left.\cdot \mathrm{h}^{\rho 3} / \mathrm{km}^{\rho 2+\rho 3}\right)$ & 1 & Scale parameter \\
\hline$\rho_{2}$ & Power term of length in revenue model & 1 & CRS of link length \\
\hline$\rho_{3}$ & Power term of speed in revenue model & 0 & Distance based only \\
\hline$\mu$ & Scale coefficient in cost model $\left(\right.$ dollar $\left.\cdot \mathrm{h}^{\alpha 2} / \mathrm{km}^{\alpha 1}\right)$ & 20 & Scale parameter \\
\hline$\alpha_{1}$ & Power term of length in cost model & 1 & CRS of link length \\
\hline$\alpha_{2}$ & Power term of capacity in cost model & 1.25 & DRS of capacity \\
\hline$\alpha_{3}$ & Power term of flow in cost model & 0 & Ignore variable cost \\
\hline$\omega_{1}, \omega_{2}$ & Coefficient in the speed-capacity log-linear regression model & $-30.6,9.8$ & Empirical estimates based on Twin Cities data \\
\hline$\eta_{0}, \eta_{1}, \eta_{2}$ & Coefficient in the capacity-number-of-lane quadratic regression model & $341,273,162$ & Empirical estimates based on Twin Cities data \\
\hline$\Phi$ & Scale coefficient in cost model & 1 & Scale parameter \\
\hline$\sigma_{1}$ & Power term of length in cost model & 1 & CRS of link length \\
\hline$\sigma_{2}$ & Power term of capacity in cost model & 1.25 & DRS of capacity \\
\hline$\sigma_{3}$ & Power term of $\Delta$ capacity in cost model & 1 & CRS in road construction \\
\hline
\end{tabular}

NOTE: CRS are DRS constant and decreasing returns to scale. 
for the discussion of benefits and costs (18). The costs to society may not be considered real costs to a company unless they are somehow internalized. Again, a public agency is considered, and it is assumed that its goal is to maximize social welfare.

The life-cycle cost $\left(C_{a}\right)$ of a road expansion project can be estimated by the construction and maintenance cost functions developed as described above:

$$
\begin{aligned}
C_{a}= & \phi \cdot\left(l_{a}\right)^{\sigma_{1}}\left(F_{a}^{0}\right)^{\sigma_{2}}\left(F_{a}^{1}-F_{a}^{0}\right)^{\sigma_{3}} \\
& +\sum_{i=1}^{y} \frac{1}{(1+R)^{y}}\left[\mu \cdot\left(l_{a}\right)^{\alpha_{1}}\left(F_{a}^{1}\right)^{\alpha_{2}}\left(f_{a}^{i}\right)^{\alpha_{3}}\right]
\end{aligned}
$$

where

$$
\begin{aligned}
y= & \text { planning horizon or estimated life duration of the road; } \\
R= & \text { interest rate; } \\
F_{a}^{0}= & \text { existing capacity; } \\
F_{a}^{1}= & \text { capacity after expansion; and } \\
f_{a}^{i}= & \text { traffic volume in year } y, \text { where } f_{a}^{i}=(1+x)^{i} f_{a}^{0}, f_{a}^{0} \text { is the } \\
& \text { existing traffic volume, and } x \text { is the annual rate of traffic } \\
& \text { growth. }
\end{aligned}
$$

The life-cycle benefits of road expansion are usually manifold, and some components are hard to quantify. Once a road is expanded, the congestion level on the road being expanded will drop, at least in the short run, resulting in less commute time, more reliable travel, fewer accidents, and reduced air pollution and energy consumption. The exact systemwide benefits are less clear because the expansion of a road tends to increase congestion on its complementary roads and attracts traffic away from its competitors. For simplicity, a formula for benefit $\left(B_{a}\right)$ estimation that considers only travel time savings on the road being expanded is specified. All nonlocal benefits, as well as influences on accidents, pollution, and fuel consumption, are ignored. Induced demand is also ignored.

$$
\begin{aligned}
B_{a}= & \sum_{i=1}^{y} \lambda \cdot \psi \cdot f_{a}^{i}\left\{\frac{l_{a}}{v_{a}^{0}}\left[1+\theta_{1}\left(\frac{f_{a}^{i}}{F_{a}^{0}}\right)^{\theta_{2}}\right]\right. \\
& \left.-\frac{l_{a}}{v_{a}^{1}}\left[1+\theta_{1}\left(\frac{f_{a}^{i}}{F_{a}^{1}}\right)^{\theta_{2}}\right]\right\}
\end{aligned}
$$

The optimal amount of capacity expansion can be identified for each road by maximizing the resulting benefit-cost ratio. This is a nonlinear programming problem with a fairly complex objective function. However, roads can be expanded by only a fixed number of lanes, which helps simplify the optimization problem. The benefit-cost ratios that correspond to the addition of one, two, or three lanes can be evaluated quickly; and the maximum possible benefit-cost ratio $\left(B C^{*}\right)$, as well as the optimal number of lanes to be expanded $\left(F^{*}\right)$, can be identified for each road. The available funds should always be allocated to expand the road with the highest $B C^{*}$ by $F^{*}$ until exhaustion of the funds.

\section{EMERGENCE OF ROAD HIERARCHY}

The network growth model can be applied to any realistic roadway network, and the execution time is largely determined by the convergence speed of the traffic assignment algorithm. A 10-by-10 grid network (100 nodes and 360 links) is used in this section to evaluate the two investment policies, especially their impacts on network reliability. The same initial condition is specified for both policy scenarios. All links in the grid network are $4 \mathrm{~km}$ in length and have an initial capacity of 735 vehicles per hour (this value corresponds to a one-lane road, according to the regression analysis with the capacity and number-of-lane data in the Twin Cities; see Equation 7). The initial network is heavily congested, with an average $S$ value of 0.8 and an average speed of about $10 \mathrm{~km} / \mathrm{h}$. The initial land use is uniform among all 100 network zones, with 10,000 trips originating from and destined for each zone. O-D demand is not fixed over the years because of changes in link capacity and real travel costs. The convergence of the simulation model can be measured directly by the number of expansion activities in the network. Under centralized control, the network achieves long-run supplydemand equilibrium if the total revenue is just equal to the total required maintenance cost (i.e., there are no more road expansions or contractions).

Network investment based on benefit-cost analysis produces a more efficient road network in the short run and the long run (Figure 3). An interesting observation is that the total number of vehicle hours of travel (VHT) in both scenarios seems to converge over time. After the equilibrium is achieved, the difference in VHT is less than $3 \%$ (benefit-cost analysis $=0.31$ million hours; bottleneck removal $=0.32$ million hours), although the largest difference over the 50 -year period is $20 \%$. The bottleneck removal policy always expands the most congested roads first, without a full assessment of benefits and costs. However, the mechanism of induced demand, sometimes depicted as the "iron law of traffic congestion" (20), starts taking effect and the expanded capacity is quickly filled by new traffic. This phenomenon has also been repeatedly observed in reality. The level of congestion on a newly expanded road can return to its original level within a short period of time $(20,21)$. As such, the roads expanded in a previous year are actually more likely to be expanded again under the bottleneck removal policy. Over time, this the-rich-get-richer cycle creates highly hierarchical road patterns (Figure 4). The distribution of road capacity seems to follow a power law (22). Similar patterns are not observed when investment decisions are guided by benefit-cost analysis. The capacity distribution may be approximated well by a normal distribution (or a Poisson distribution, because of nonnegativity), although there are not enough data points to verify this hypothesis. The real capacity distribution of all roads in the Twin Cities network in 1998 suggests that it is probably an investment policy closer to bottleneck removal that

\section{VHT (million hours)}

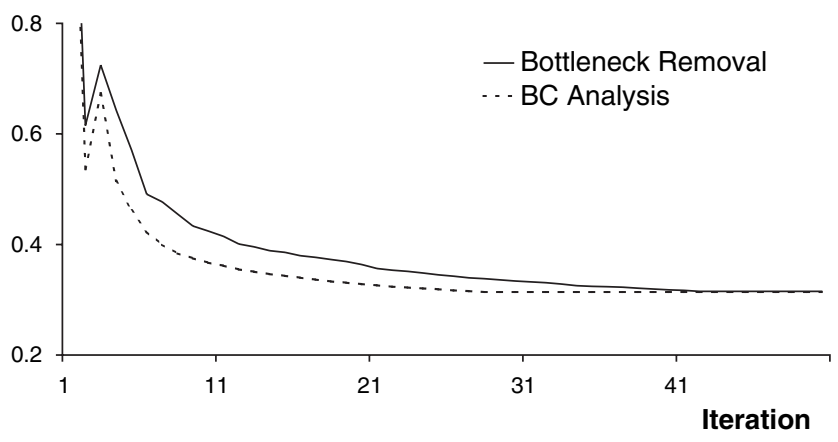

FIGURE 3 Network efficiency under alternative investment policies. 


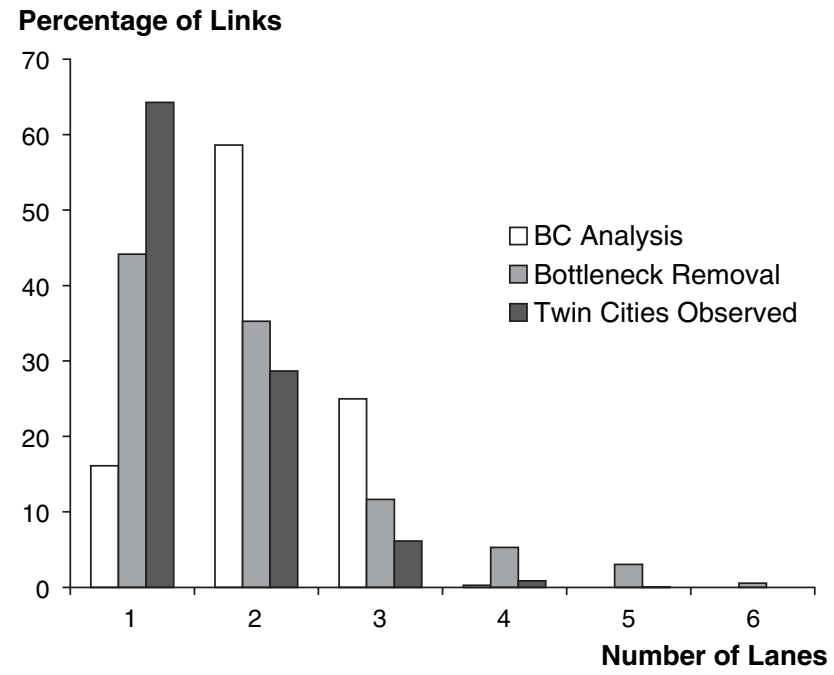

FIGURE 4 Distributions of road capacity.

has historically driven the growth of the network in the region. The distinct growth paths under the two investment policies can be seen more vividly in Figure 5.

Traditionally, a network hierarchy is concerned only with how nodes are connected to each other, whereas link properties, such as capacity and the connection cost, are ignored. The ignoring of such properties may not be a serious problem for some networks on which nodes are the most important agents in the system [e.g., social networks (23) and the World Wide Web (24)]. However, in transportation networks, especially road networks, link properties are of paramount importance because they largely determine network performance. Therefore, the hierarchy in road networks has at least two dimensions: the connectivity hierarchy and the capacity hierarchy. A star network (i.e., a perfect hub-and-spoke system) has the highest connectivity hierarchy. A network with a link capacity distributed according to the power law has the highest-capacity hierarchy. The discussion about the emergence of a road hierarchy in the simulation experiment presented above considers only the capacity hierarchy under the two investment policies, because the connectivity hierarchy is equal by design.

\section{NETWORK FRAGILITY AND VULNERABILITY}

The simulated network growth process has many interesting properties. However, the focus of this research is the relationship between investment policies, road hierarchy, network fragility, and vulnerability. Does the more hierarchical road arrangement induced by the bottleneck removal policy have a better failure tolerance than the flatter network from the benefit-cost analysis? A Monte Carlo simulation method was adopted to shed light on this reliability issue. In each run of the simulation, a certain percentage of links in the equilibrium networks under the two investment policies are removed on the basis of one of three criteria, and the performance characteristics of the degraded networks are assessed. Travel demand is reassigned to the remaining links but is not redistributed between O-D pairs. The assumption here is that capacity loss because of the various types of road failures described below is not long enough to cause significant demand responses other than rerouting.
The three network failure scenarios considered are $(a)$ a random link failure, in which the probability that a link loses its flow-carrying function is purely random (Scenario 1); (b) a volume-dependent failure, in which a link carrying more traffic is more likely to break down than its low-volume peers and the failure rate is proportional to the traffic volume (Scenario 2); and (c) the most important links, defined as those with the highest capacity, are destroyed by deliberate attacks (Scenario 3). Scenario 1 corresponds well to various types of non-traffic-related road malfunctioning, including natural disasters. Scenario 2 tries to capture the impacts of link breakdowns caused by traffic-related incidents, such as accidents and routine maintenance. The presumed linear relationship between accident rates and traffic volume is only an approximation and has been supported by some empirical studies performed with aggregated traffic data. More recent studies controlling for factors such as speed, weaving flow, weather, and lighting conditions (25-28) conclude that the true relationships between accident rates and traffic flow are more complicated. The level of congestion, often measured by the determination of $S$, also contributes to the variation in accident rates in a nonlinear manner (29). Better models of traffic-related link failure may be adopted in future research. Scenario 3 tests the serviceability of a road network in the case of targeted attacks. A Monte Carlo simulation is not required for Scenario 3, for obvious reasons. A network is considered fragile if it performs poorly with even a small number of random or volume-dependent link failures. On the other hand, if a network deteriorates quickly when its most important links are removed, it is said to be vulnerable.

The flatter road network under the benefit-cost investment rule displays advantages in all three link failure scenarios (Figure 6). Each dot in the graph represents the average performance of 30 Monte Carlo simulation runs. It was decided that 30 runs would be sufficient because the Markovian variation of the average performance is negligible. The smallest performance differences between the two networks are observed with random failure rates, and large differences are observed under targeted attacks. In each one of the three scenarios, the superiority of the flatter network becomes more obvious as more links are removed. The highly hierarchical network created by the bottleneck removal policy is extremely vulnerable. The total travel time increases exponentially as more and more top links are destroyed. In particular, the total travel time doubles when only $4 \%$ of top links (14 of 360) are removed. This finding is consistent with the identified vulnerability of other scale-free or power law networks (24). When the performance measure is plotted for each network separately, it is found that the less hierarchical network exhibits slow increases in travel costs in all types of network degradation (Figure 7). The relationship between the percentage of removed links and the percent increase in the total travel time is best described by a linear function. In the network with a higher degree of road hierarchy, nonlinear increases in travel time are evident. In both networks, random link failure is the least serious kind of network deterioration and targeted attacks result in the largest loss of efficiency, a result that is quite intuitive.

\section{CONCLUSIONS}

Models of network growth and policies are able to demonstrate how the characteristics of investment rules shape the network form, create various degrees of road hierarchy, and determine the performance of 


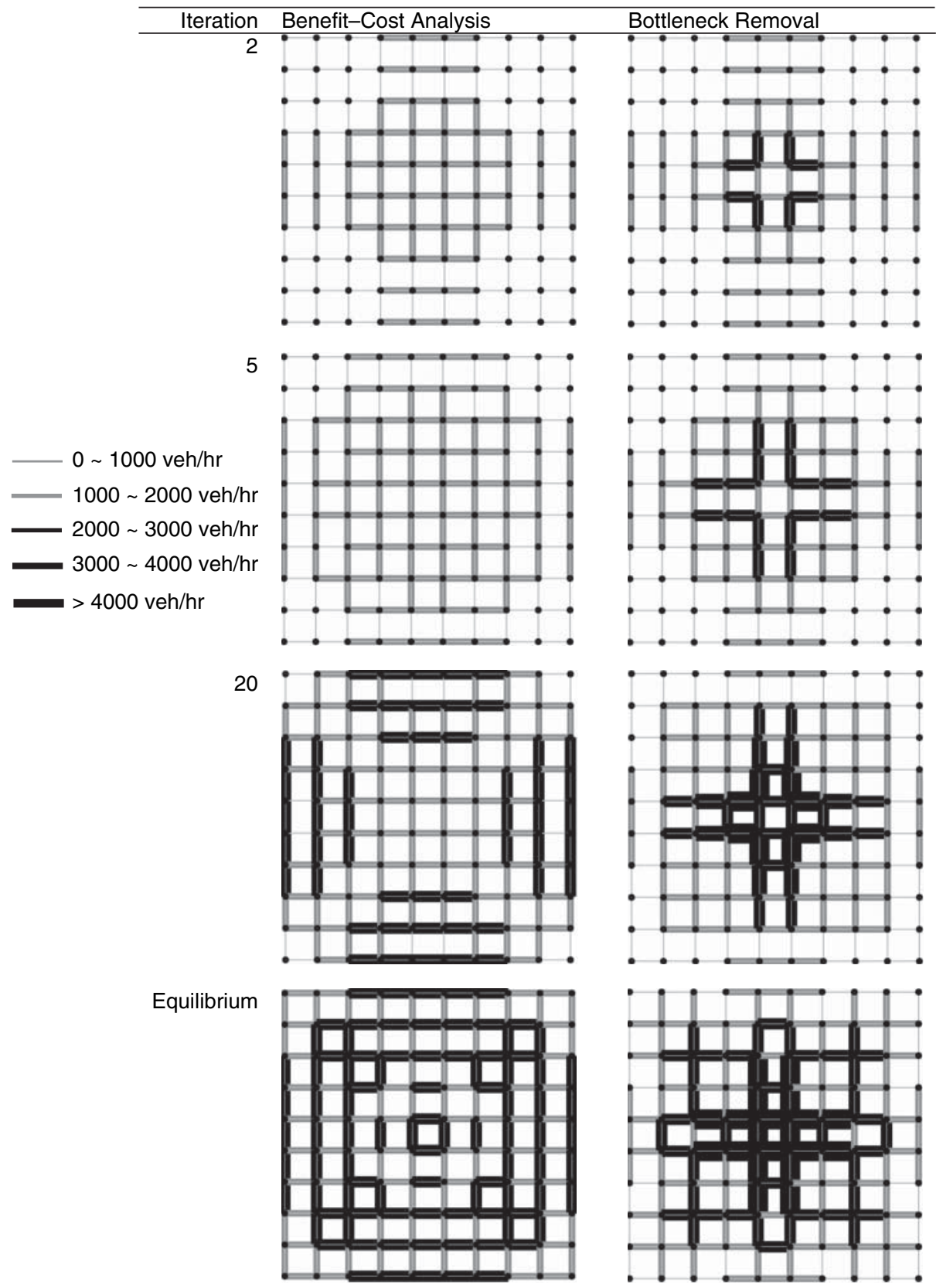

FIGURE 5 Network evolution under alternative investment policies.

the network under normal and degraded conditions. Investment based on benefit-cost analysis leads to a less hierarchical network structure than the more myopic bottleneck removal program. In general, a highly hierarchical network structure displays moderate increases in travel cost when link failure rates are random or volume dependent, but the performance of the network deteriorates at an exponential rate as top links are removed in the case of a targeted attack. In this sense, a road network with a capacity distributed according to the power law is slightly fragile but extremely vulnerable. In contrast, a flatter network, such as the one stemming from repetitive benefit-cost analyses, is neither fragile nor vulnerable. It should be pointed out that the results also do not suggest a zero-hierarchy network. Hierarchy exists in road networks for reasons such as economic efficiency, but an overly hierarchical structure has serious reliability problems (when both fragility and vulnerability are considered).

These findings have several policy implications. Throughout the equilibration or evolution process, the network studied under benefit-cost analysis has better efficiency performance and error and attack tolerance. Therefore, benefit-cost analysis should be preferred to the myopic bottleneck removal type of investment rules, no matter how the planning horizon is specified. A trade-off between network efficiency and reliability is not present in the grid network being analyzed, but such a trade-off may be identified when other sets of policies are compared. 


\section{Percentage Increase of VHT}

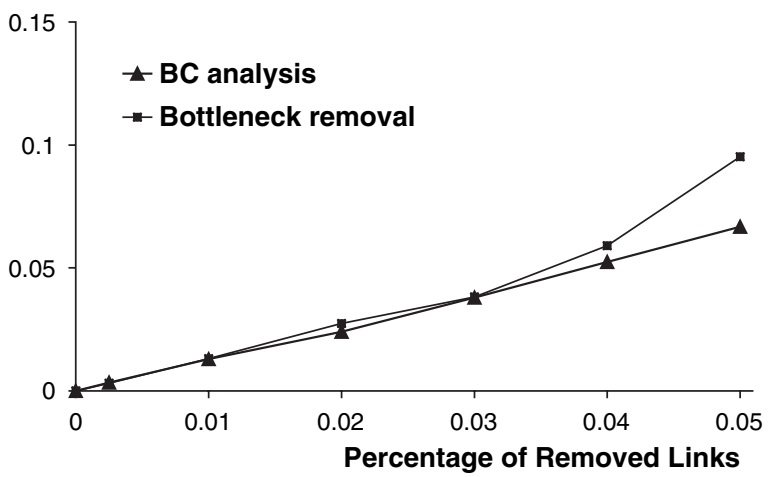

(a)

\section{Percentage Increase of VHT}

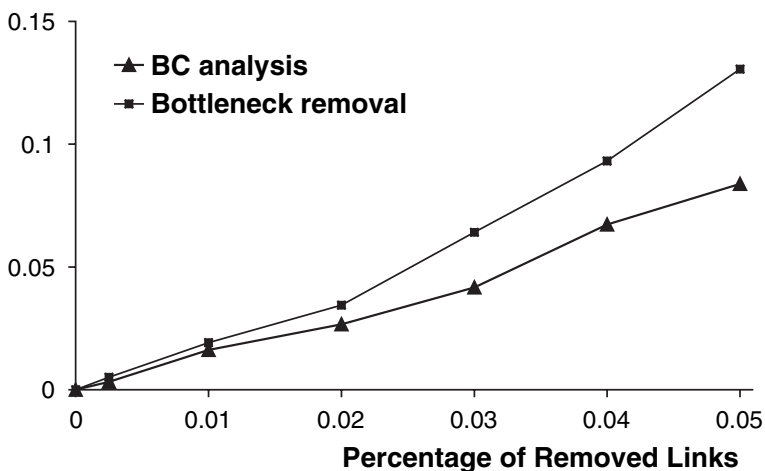

(b)

\section{Percentage Increase of VHT}

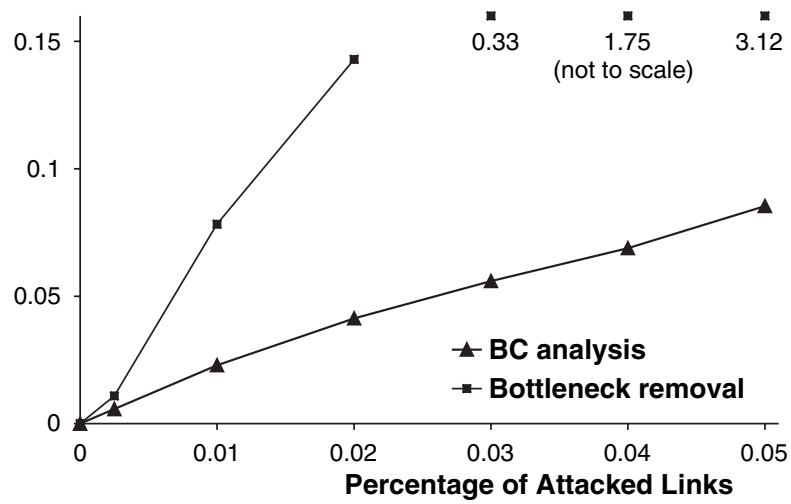

(c)

FIGURE 6 Network fragility and vulnerability, between-network comparison: (a) random link failure, $(b)$ volume-dependent failure, and $(c)$ targeted attack.

Besides investment rules, revenue mechanisms (e.g., pricing policies) and ownership structures (e.g., public or private) are also controllable factors that influence the shape of road networks. The method described here may also be applied to assessment of the long-run efficiency and reliability of a road network under any combination of road financing policies. Application to a realistic road network would also be interesting and should have immediate practical significance.
Percentage Increase of VHT

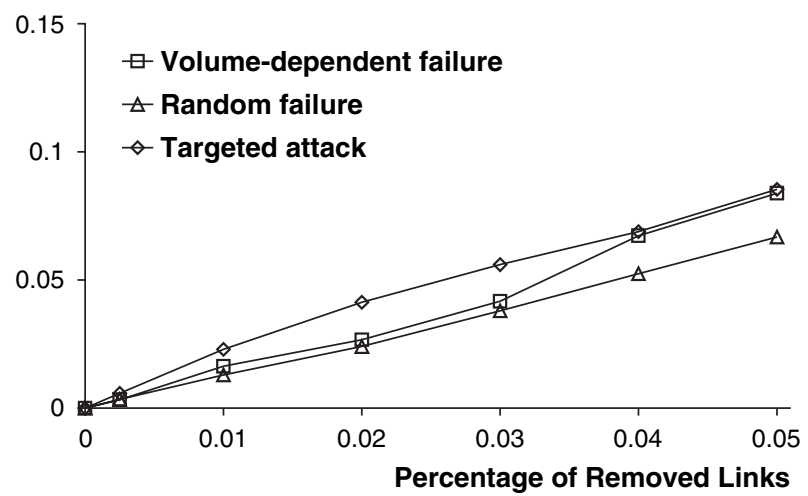

(a)

\section{Percentage Increase of VHT}

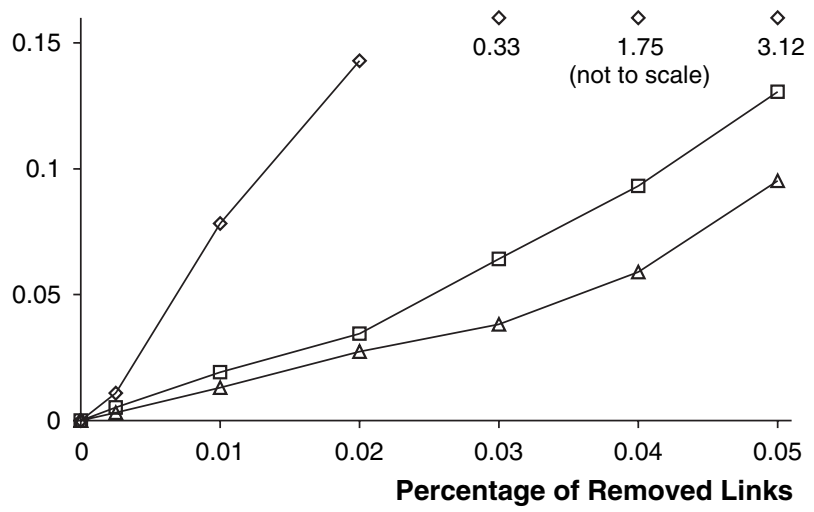

(b)

FIGURE 7 Network fragility and vulnerability, within-network comparison: $(a)$ benefit-cost analysis and $(b)$ bottleneck removal.

\section{REFERENCES}

1. Iida, Y., and H. Wakabayashi. An Approximation Method of Terminal Reliability of a Road Network Using Partial Minimal Path and Cut Set. Proc., 5th World Conference on Transport Research, Yokohama, Japan, 1989, pp. 367-380.

2. Asakura, Y., and M. Kashiwadani. Road Network Reliability Caused by Daily Fluctuation of Traffic Flow. Proc., 19th PTRC Summer Annual Meeting, Brighton, U.K., PTRC, London, 1991, pp. 73-84.

3. Bell, M. G. H., C. Cassir, Y. Iida, and W. H. K. Lam. A Sensitivity Based Approach to Reliability Assessment. Proc., 14th International Symposium on Transportation and Traffic Theory, Pergamon, New York, 1999, pp. 283-300.

4. Chen, A., H. Yang, H. K. Lo, and W. Tang. A Capacity Related Reliability for Transportation Networks. Journal of Advanced Transportation, Vol., 33, No. 2, 1999, pp. 183-200.

5. Lam, W. H. K., and N. Zhang. A New Concept of Travel Demand Satisfaction Reliability for Assessing Road Network Performance. Presented at the Matsuyama Workshop on Transport Network Analysis, Matsuyama, Japan, Aug. 2000.

6. D'Este, G. M., and M. A. P. Taylor. Network Vulnerability: An Approach to Reliability Analysis at the Level of National Strategic Transport Networks. In The Network Reliability of Transport (M. G. H. Bell and Y. Iida, eds.), Elsevier Science, Oxford, United Kingdom, 2003.

7. Asakura, Y., E. Hato, and M. Kashiwadani. Stochastic Network Design Problem: An Optimal Link Investment Model for Reliable Network. In The Network Reliability of Transport (M. G. H. Bell and Y. Iida, eds.), Elsevier Science, Oxford, United Kingdom, 2003.

8. Haynes, K. E., and A. S. Fotheringham. Gravity and Spatial Interaction Models. Sage Publications, Beverly Hills, Calif., 1984. 
9. Bar-Gera, H., and D. Boyce. Origin-Based Algorithm for Combined Travel Forecasting Models. Transportation Research, Part B, Vol. 37, No. 5, 2003, pp. 405-422.

10. Bureau of Public Roads. Traffic Assignment Manual. Urban Planning Division, U.S. Department of Commerce, Washington, D.C., 1964.

11. Roth, G. Roads in a Market Economy. Avebury Technical, Aldershot, England, 1996.

12. Keeler, T. E., and K. A. Small. Optimal Peak-Load Pricing, Investment, and Service Levels on Urban Expressways. Journal of Political Economy, Vol. 85, No. 1, 1977, pp. 1-26.

13. Levinson, D., and R. Karamalaputi. Induced Supply: A Model of Highway Network Expansion at the Microscopic Level. Journal of Transportation Economics and Policy, Vol. 37, No. 3, 2003, pp. 297-318.

14. Gómez-Ibáñez, J. A. Pricing. In Essays in Transportation Economics and Policy: A Handbook in Honor of John R. Meyer (J. A. Gómez-Ibáñez, W. B. Tye, and C. Winston, eds.), Brookings Institution Press, Washington, D.C., 1999 , p. 121.

15. Heggie, I. G. Management and Financing of Roads: An Agenda for Reform. Working Paper No. 8. Sub-Sahara Africa Transport Policy Program, World Bank, Washington, D.C., 1995.

16. Kraus, M. Scale Economies Analysis for Urban Highway Networks. Journal of Urban Economics, Vol. 9, 1981, p. 1.

17. Meyer, J., J. Kain, and M. Wohl. The Urban Transportation Problem. Harvard University Press, Cambridge, Mass., 1965.

18. Wohl, M., and C. Hendrickson. Transportation Investment and Pricing Principles. John Wiley and Sons, Inc., New York, 1984.

19. Downs, A. The Law of Peak-Hour Expressway Congestion. Traffic Quarterly, Vol. 16, No. 3, 1962, pp. 393-409.

20. Goodwin, P. B. Empirical Evidence on Induced Traffic: A Review and Synthesis. Transportation, Vol. 23, 1996, pp. 35-54.
21. Parthasarathi, P., D. Levinson, and R. Karamalaputi. Induced Demand: A Microscopic Perspective. Urban Studies, Vol. 40, No. 7, 2003 pp. $1335-1353$.

22. Zipf, G. K. Human Behavior and the Principles of Least Effort. Addison Wesley, Cambridge, Mass., 1949.

23. Granovetter, M. Getting a Job: A Study of Contacts and Careers. University of Chicago Press, Chicago, Ill., 1995.

24. Albert, R., H. Jeong, and A. L. Barabási. Attack and Error Tolerance in Complex Networks. Nature, Vol. 406, 2000, p. 378.

25. Aljanahi, A. A. M., A. H. Rhodes, and A. V. Metcalfe. Speed, Speed Limits and Road Traffic Accidents Under Free Flow Conditions. Accident Analysis and Prevention, Vol. 31, 1999, pp. 161-168.

26. Cedar, A., and L. Livneh. Relationship Between Road Accidents and Hourly Traffic Flow. Accident Analysis and Prevention, Vol. 14, 1982, pp. 19-44.

27. Dickerson, A., J. Peirson, and R. Vickerman. Road Accidents and Traffic Flows: An Econometric Investigation. Economica, Vol. 67 2000, pp. 101-121.

28. Golob, T. F., and W. W. Recker. Relationships Among Urban Freeway Accidents, Traffic Flow, Weather, and Lighting Conditions. Journal of Transportation Engineering, Vol. 129, No. 4, 2003, pp. 342-353.

29. Zhou, M., and V. P. Sisiopiku. Relationship Between Volume-toCapacity Ratios and Accident Rates. In Transportation Research Record 1581, TRB, National Research Council, Washington, D.C., 1997, pp. 47-52.

The Critical Transportation Infrastructure Protection Committee sponsored publication of this paper. 